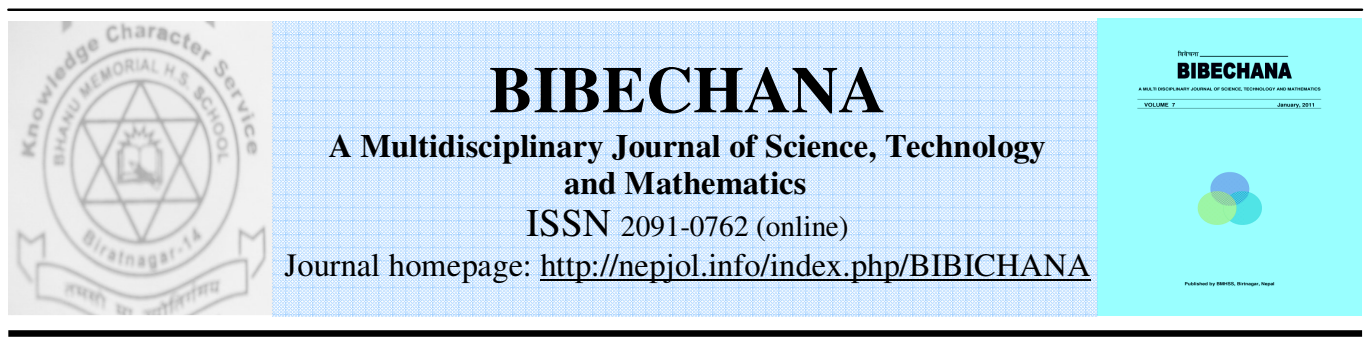

\title{
Applications of KS - variables in celestial mechanics
}

\author{
M.R. Hassan ${ }^{a}$, R.R. Thapa ${ }^{b}$ * \\ ${ }^{a}$ Dept. of Mathematics, S.M. College, Bhagalpur, India \\ ${ }^{\mathrm{b}}$ Dept. of Mathematics, P.G. College, Biratnagar, Morang, Nepal \\ Article History: Received 5 September 2010; Revised 18 November 2010; Accepted 29 November 2010
}

Abstract

This paper deals with the transformation of KS-Variables and canonically conjugate variables from sideral (inertial) to synodic (rotating) form and their applications in "the circular restricted problem of three bodies in three-dimensional coordinate system" to form generating solutions.

\section{Keywords : KS - transformation; KS - Variables; Orbital elements; Generating solutions}

\section{Introduction}

Kustannheimo and Steifel [1] presented a very convenient regularization method for the space motion of particles near an attracting centre. This mechanism leads to a transformation, called KS - transformation. The transformation is of the type

$$
\mathrm{x}_{\mathrm{k}}=\mathrm{x}_{\mathrm{k}}\left(\mathrm{u}_{\mathrm{j}}\right),(\mathrm{k}=1,2, \ldots \ldots \ldots \mathrm{n}),(\mathrm{j}=1,2,3, \ldots \ldots ., \mathrm{n}+1)
$$

which increases the number of variables. In this regard Schiefle [2] and Steifel and Schiefle [3] have considered the so - called generalized canonical transformation increasing the number of variables.

As in physical space, $\mathrm{KS}$ - regularization is the most perfect regularization, so to solve all the problems easily of three dimensional synodic coordinate system by KS - Variables; it is necessary to transform the KS - Variables from sidereal to synodic form. In this paper, we attempted to present the KS - Variables in synodic form and their simple application to the motion of an infinitesimal mass in the restricted problem of the three bodies in three dimensional synodic coordinate system have been shown.

In earlier sections we just presented some important tools of KS - Transformations in terms of orbital elements $\mathrm{i}, \Omega, \omega, \sigma, \mathrm{t}$ etc. in synodic coordinate system. Some related and conjugate variables are also presented here in these sections. In later section, the synodic KS Variables are used to solve the equations of motion of infinitesimal mass in the circular restricted three - body problem in three dimensional synodic coordinate system in the form of generating solutions.

The symbols used are as follows:

$\mathrm{i}=$ inclination of the orbital plane with the plane of motion of the central body,

$\Omega=$ longitude of the ascending node,

$\omega=$ argument of perihelion,

$\phi=$ true anomaly,

$\mathrm{e}=$ eccentric anomaly,

$\mathrm{t}=$ physical time,

\footnotetext{
* Corresponding author: Raju Ram Thapa, Post Graduate Campus, Biratnagar, Tribhuvan University, Nepal, Email:thaparajuram@yahoo.com
} 


$$
\begin{aligned}
& \mathrm{s}=\text { psedo time }, \\
& \mathrm{n}=\text { mean angular motion }, .=\frac{d}{d t}, /=\frac{d}{d s}, \sigma=\phi+\omega
\end{aligned}
$$

\section{Derivation of synodic KS - Variables}

In 1965 Kustaanheimo and Steifel [1] defined an orthogonal matrix L (u), called KS - matrix, where

$$
\begin{aligned}
& \mathrm{L}(\mathrm{u})=\left[\begin{array}{cccc}
u_{1} & -u_{2} & -u_{3} & u_{4} \\
u_{2} & u_{1} & -u_{4} & -u_{3} \\
u_{3} & u_{4} & u_{1} & u_{2} \\
u_{4} & -u_{3} & u_{2} & -u_{1}
\end{array}\right] \\
& \mathrm{L}^{\mathrm{T}}(\mathrm{u}) \mathrm{L}(\mathrm{u})=\mathrm{L}(\mathrm{u}) \mathrm{L}^{\mathrm{T}}(\mathrm{u})=(\mathrm{u}, \mathrm{u}) \mathrm{I} .
\end{aligned}
$$

Here $\mathrm{L}^{\mathrm{T}}(\mathrm{u})$ is the transpose of $\mathrm{L}(\mathrm{u}), \mathrm{I}$ is the $4 \times 4$ unit matrix and $\mathrm{u}=\left(\mathrm{u}_{1}, \mathrm{u}_{2}, \mathrm{u}_{3}, \mathrm{u}_{4}\right)$.

If $\mathrm{x}=\left(\mathrm{x}_{1}, \mathrm{x}_{2}, \mathrm{x}_{3}\right)$ are three dimensional coordinates of a point, $\mathrm{KS}-$ transformation in sidereal frame is given by the single matrix equation:

$$
\mathrm{x}=\mathrm{L}(\mathrm{u})(\mathrm{u})
$$

$$
\begin{array}{llll}
\text { Explicitly, } & \mathrm{x}_{1} & = & \mathrm{u}_{1}{ }^{2}-\mathrm{u}_{2}{ }^{2}-\mathrm{u}_{3}{ }^{2}+\mathrm{u}_{4}{ }^{2} \\
& \mathrm{x}_{2} & = & 2\left(\mathrm{u}_{1} \mathrm{u}_{2}-\mathrm{u}_{3} \mathrm{u}_{4}\right) \\
& \mathrm{x}_{3} & = & 2\left(\mathrm{u}_{1} \mathrm{u}_{3}-\mathrm{u}_{2} \mathrm{u}_{3}\right)
\end{array}
$$

In sidereal frame, the compact parametric representation of $\mathrm{KS}-$ Variables in terms of orbital elements i, $\Omega, \sigma$ in any plane curve having its orbital plane in general position, is given by [3] as :

$$
\begin{array}{rlrl}
\mathrm{u}_{1} & =\sin \left(\frac{i}{2}\right) \cos \left[\frac{(\Omega-\sigma)}{2}\right] & u_{2}=\sin \left(\frac{i}{2}\right) \sin \left[\frac{(\Omega-\sigma)}{2}\right] \\
\mathrm{u}_{3}=\cos \left(\frac{i}{2}\right) \sin \left[\frac{(\Omega+\sigma)}{2}\right] & u_{4}=-\cos \left(\frac{i}{2}\right) \cos \left[\frac{(\Omega+\sigma)}{2}\right]
\end{array}
$$

The sidereal parameters $u_{j}(j=1,2,3,4)$ of the particle itself are obtained by multiplying the square root of its distance ' $r$ ' from the central body.

Therefore,

$$
\begin{array}{ll}
\mathrm{u}_{1}=\sqrt{r} \sin \left(\frac{i}{2}\right) \cos \left[\frac{(\Omega-\sigma)}{2}\right] & \mathrm{u}_{2}=\sqrt{r} \sin \left(\frac{i}{2}\right) \sin \left[\frac{(\Omega-\sigma)}{2}\right] \\
\mathrm{u}_{3}=\sqrt{ } r \cos \left(\frac{i}{2}\right) \sin \left[\frac{(\Omega+\sigma)}{2}\right] & \mathrm{u}_{4}=-\sqrt{r} r \cos \left(\frac{i}{2}\right) \cos \left[\frac{(\Omega+\sigma)}{2}\right]
\end{array}
$$

Where $\mathrm{r}=\left(\mathrm{x}_{1}^{2}+\mathrm{x}_{2}^{2}+\mathrm{x}_{3}\right)^{1 / 2}=\mathrm{u}_{1}^{2}+\mathrm{u}_{2}^{2}+\mathrm{u}_{3}^{2}+\mathrm{u}_{4}{ }^{2}=(\mathrm{u}, \mathrm{u})$

It may be noted that i \& $\Omega$ are fixed orbital elements whereas $\sigma$ is only the variable orbital element.

In terms of orbital elements $\mathrm{i}, \Omega \& \sigma$ the sidereal physical coordinates $\mathrm{x}_{\mathrm{i}}^{\mathrm{s}}$ of a point are given by

$$
\begin{aligned}
& \mathrm{x}_{1}=\sin ^{2}\left(\frac{i}{2}\right) \cos (\Omega-\sigma)+\cos ^{2}\left(\frac{i}{2}\right) \cos (\Omega+\sigma) \\
& \mathrm{x}_{2}=\sin ^{2}\left(\frac{i}{2}\right) \sin (\Omega-\sigma)+\cos ^{2}\left(\frac{i}{2}\right) \sin (\Omega+\sigma) \\
& \mathrm{x}_{3}=\sin \text { i. } \sin \sigma
\end{aligned}
$$

If $\mathrm{x}_{1} \mathrm{x}_{2}$ plane rotates about $\mathrm{x}_{3}$ - axis with mean angular motion $\mathrm{n}$, then at any time $\mathrm{t}$, the synodic coordinates $\left(\mathrm{y}_{1}, \mathrm{y}_{2}, \mathrm{y}_{3}\right)$ of the point, are given by

$$
\mathrm{y}_{1}=\mathrm{x}_{1} \cos \mathrm{nt}+\mathrm{x}_{2} \sin \mathrm{nt}, \mathrm{y}_{2}=-\mathrm{x}_{1} \sin \mathrm{nt}+\mathrm{x}_{2} \cos \mathrm{nt}, \mathrm{y}_{3}=\mathrm{x}_{3}
$$

Introduction of Eqs. (7) in (8) yields 


$$
\begin{aligned}
& \mathrm{y}_{1}=\sin ^{2}\left(\frac{i}{2}\right) \cos (\Omega-\sigma-n t)+\cos ^{2}\left(\frac{i}{2}\right) \cos (\Omega+\sigma-n t) \\
& \mathrm{y}_{2}=\sin ^{2}\left(\frac{i}{2}\right) \sin (\Omega-\sigma-n t)+\cos ^{2}\left(\frac{i}{2}\right) \sin (\Omega+\sigma-n t) \\
& \mathrm{y}_{3}=\sin \text { i. } \sin \sigma
\end{aligned}
$$

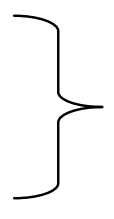

If $\mathrm{q}=\left(\mathrm{q}_{1}, \mathrm{q}_{2}, \mathrm{q}_{3}, \mathrm{q}_{4}\right)$ are the synodic $\mathrm{KS}-$ Variables corresponding to physical synodic coordinates $\mathrm{y}=\left(\mathrm{y}_{1}, \mathrm{y}_{2}, \mathrm{y}_{3}\right)$, then

Explicitly:

$$
\mathrm{Y}=\mathrm{L}(\mathrm{q}) \mathrm{q}
$$

$$
\mathrm{y}_{1}=\mathrm{q}_{1}^{2}-\mathrm{q}_{2}^{2}-\mathrm{q}_{3}^{2}+\mathrm{q}_{4}^{2}, \quad \mathrm{y}_{2}=2\left(\mathrm{q}_{1} \mathrm{q}_{2}-\mathrm{q}_{3} \mathrm{q}_{4}\right), \quad \mathrm{y}_{3}=2\left(\mathrm{q}_{1} \mathrm{q}_{3}+\mathrm{q}_{2} \mathrm{q}_{4}\right)
$$

From Eqs. (9) \& (11) the parametric representation of a point in synodic KS - Variables are given

$$
\begin{array}{ll}
\mathrm{q}_{1}=\sin \left(\frac{i}{2}\right) \cos \left[\frac{(\Omega-\sigma-n t)}{2}\right] & \mathrm{q}_{2}=\sin \left(\frac{i}{2}\right) \sin \left[\frac{(\Omega-\sigma-n t)}{2}\right] \\
\mathrm{q}_{3}=\cos \left(\frac{i}{2}\right) \sin \left[\frac{(\Omega+\sigma-n t)}{2}\right] & \mathrm{q}_{4}=-\cos \left(\frac{i}{2}\right) \cos \left[\frac{(\Omega+\sigma-n t)}{2}\right]
\end{array}
$$

Which satisfy all the Eqs.(9) \& (11) \& properties of KS - Transformations.

The synodic parameters $q_{j}(j=1,2,3,4)$ of the particle itself can be obtained by multiplying by the square root of its distance ' $\mathrm{r}$ ' from the central body.

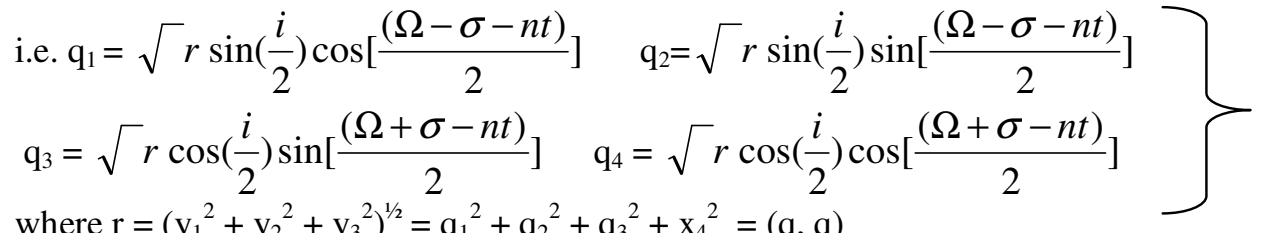

From symbolic representations, we have $\sigma=\omega+\phi$ where $\omega$ is the constant orbital element. i.e.

$\dot{\sigma}=\dot{\phi}$

\section{Derivation of the generalized momenta corresponding to synodic KS - Variables}

The set of synodic KS - Variables given in Eq.(13) acquire great importance, while studying the motion of an infinitesimal mass in synodic frame of reference. Moreover, in Hamiltonian dynamics, the generalized momenta corresponding to the generalized coordinates are also of great importance, so here we need to establish the generalized momenta corresponding to the synodic $\mathrm{KS}-$ Variables in terms of orbital elements $\mathrm{i}, \Omega, \sigma$ etc.

If $\mathrm{X}=\left(\mathrm{X}_{1}, \mathrm{X}_{2}, \mathrm{X}_{3}\right)$ are the momenta corresponding to sidereal physical coordinates $\mathrm{x}=$ $\left(\mathrm{x}_{1}, \mathrm{x}_{2}, \mathrm{x}_{3}\right)$ and $\mathrm{P}=\left(\mathrm{P}_{1}, \mathrm{P}_{2}, \mathrm{P}_{3}, \mathrm{P}_{4}\right)$ are the generalized momenta corresponding to sidereal parametric coordinates $\mathrm{u}=\left(\begin{array}{llll}\mathrm{u}_{1} & \mathrm{u}_{2} & \mathrm{u}_{3} & \mathrm{u}_{4}\end{array}\right)$, then momenta transformation is given by the single matrix equation:

Explicitly

$$
\left.\begin{array}{l}
x=(1 / 2 r) L(u) P \\
X_{1}=\frac{\left(P_{1} u_{1}-P_{2} u_{2}-P_{3} u_{3}+P_{4} u_{4}\right)}{2 r} \\
X_{2}=\frac{\left(P_{1} u_{2}+P_{2} u_{1}-P_{3} u_{4}-P_{4} u_{3}\right)}{2 r} \\
X_{3}=\frac{\left(P_{1} u_{3}+P_{2} u_{4}+P_{3} u_{1}+P_{4} u_{2}\right)}{2 r}
\end{array}\right\}
$$

The bilinear relation (16) plays an important key role in the above representations. The inverse transformation of (15) is given as :

$$
\mathrm{P}=2 \mathrm{H}(\mathrm{u}) \mathrm{X}
$$


Explicitly from Eqs. (3) and (15)

$$
\begin{array}{ll}
\mathrm{P}_{1}=2\left(\mathrm{X}_{1} \mathrm{u}_{1}+\mathrm{X}_{2} \mathrm{u}_{2}+\mathrm{X}_{3} \mathrm{u}_{3}\right) & \mathrm{P}_{2}=2\left(-\mathrm{X}_{1} \mathrm{u}_{2}+\mathrm{X}_{2} \mathrm{u}_{1}+\mathrm{X}_{3} \mathrm{u}_{4}\right) \\
\mathrm{P}_{3}=2\left(-\mathrm{X}_{1} \mathrm{u}_{3}+\mathrm{X}_{2} \mathrm{u}_{4}+\mathrm{X}_{3} \mathrm{u}_{1}\right) & \mathrm{P}_{4}=2\left(\mathrm{X}_{1} \mathrm{u}_{4}-\mathrm{X}_{2} \mathrm{u}_{3}+\mathrm{X}_{3} \mathrm{u}_{2}\right)
\end{array}
$$

Suppose that $\mathrm{Y}=\left(\mathrm{Y}_{1}, \mathrm{Y}_{2}, \mathrm{Y}_{3}\right)$ are the momenta is synodic system corresponding to $\mathrm{y}=\left(\mathrm{y}_{1}, \mathrm{y}_{2}, \mathrm{y}_{3}\right)$, then from the definition of momenta

$$
\mathrm{Y}_{1}=\dot{y_{1}}-n y_{2}, \mathrm{Y}_{2}=\dot{y_{2}}+n y_{1}+\mathrm{ny}_{1}, \mathrm{Y}_{3}=\dot{y_{3}}
$$

From Eqs. (7) and (20) we get

$$
\left.\begin{array}{l}
\mathrm{Y}_{1}=\dot{\sigma}\left[\sin ^{2}\left(\frac{i}{2}\right) \sin (\Omega-\sigma-n t)-\cos ^{2}\left(\frac{i}{2}\right) \sin (\Omega+\sigma-n t)\right. \\
\mathrm{Y}_{2}=\dot{\sigma} \cdot\left[\sin ^{2}\left(\frac{i}{2}\right) \cos (\Omega-\sigma-n t)+\cos ^{2}\left(\frac{i}{2}\right) \cos (\Omega+\sigma-n t)\right.
\end{array}\right\}
$$

If $\mathrm{Q}=\left(\mathrm{Q}_{1}, \mathrm{Q}_{2}, \mathrm{Q}_{3}, \mathrm{Q}_{4}\right)$ are the generalized momenta in synodic system corresponding to $\mathrm{q}=\left(\mathrm{q}_{1}\right.$, $\left.\mathrm{q}_{2}, \mathrm{q}_{3}, \mathrm{q}_{4}\right)$, then with the help of Eq.(18), the value of $\mathrm{Q}_{\mathrm{j}}^{\mathrm{s}}$ may be represented as :

$$
\mathrm{Q}=2 \mathrm{H}(\mathrm{q}) \mathrm{Y}
$$

Therefore, $\mathrm{Q}_{1}=2\left(\mathrm{Y}_{1} \mathrm{q}_{1}+\mathrm{Y}_{2} \mathrm{q}_{2}+\mathrm{Y}_{3} \mathrm{q}_{3}\right)$

$$
=2 \dot{\sigma} \sqrt{ } r \sin \left(\frac{i}{2}\right) \sin \left[\frac{(\Omega-\sigma-n t)}{2}\right]=2 \dot{\sigma} \mathrm{q}_{2}
$$

Therefore, $\mathrm{Q}_{1}=2 \dot{\sigma} \mathrm{q}_{2}, \mathrm{Q}_{2}=-2 \dot{\sigma} \mathrm{q}_{1}, \mathrm{Q}_{3}=-2 \dot{\sigma} \mathrm{q}_{4}, \mathrm{Q}_{4}=2 \dot{\sigma} \mathrm{q}_{3}$

The set of variables $Q_{j}{ }^{s}$ and $q_{j}{ }^{s}$ satisfy the bilinear relation

$$
T(Q, q)=Q_{1} q_{4}-Q_{2} q_{3}+Q_{3} q_{2}-Q_{4} q_{1}=o
$$

Thus in both the systems $\mathrm{T}(\mathrm{P}, \mathrm{u})$ and $\mathrm{T}(\mathrm{Q}, \mathrm{q})$ are invariant.

$$
\text { Also }|\mathrm{Q}|^{2}=(\mathrm{Q}, \mathrm{Q})=\sum_{j=1}^{4} Q_{j}^{2}=4 r \dot{\sigma}^{2}
$$

\section{The equations of motion}

The regularized canonical equations of motion of the infinitesimal mass in synodic coordinate system given by Kurcheeva [4] are :

$$
\frac{d q_{j}}{d s}=\frac{\partial K}{\partial Q_{j}}, \quad \frac{d Q_{j}}{d s}=\frac{-\partial K}{\partial q_{j}} \quad(j=1,2,3,4)
$$

where the Hamiltonian $\mathrm{K}$ (regularized at $\mathrm{r}=0$ only) is given by :

pimary.

$$
\begin{aligned}
\mathrm{K}=1 / 2 & \sum_{j=1}^{4} Q^{2}{ }_{j}+2 \rho^{2}\left(Q_{1} q_{2}-Q_{2} q_{1}+Q_{3} q_{4}-Q_{4} q_{3}+C_{0}\right)-4 \\
& +4 \mu\left[1-\rho^{2}\left(\mathrm{q}_{1}{ }^{2}-\mathrm{q}_{2}{ }^{2}-\mathrm{q}_{3}{ }^{2}+\mathrm{q}_{4}{ }^{2}+\frac{1}{r_{1}}-\frac{1}{2}\left(\mathrm{c}_{1}+1\right)\right]\right.
\end{aligned}
$$

With

$$
\begin{aligned}
\mathrm{r} & =\mathrm{q}_{1}{ }^{2}+\mathrm{q}_{2}{ }^{2}+\mathrm{q}_{3}{ }^{2}+\mathrm{q}_{4}{ }^{2}=\rho^{2} \\
& =\text { the distance of the infinitesimal mass from the first primary, } \\
\mathrm{r}_{1}{ }^{2} & =1+2\left(\mathrm{q}_{1}{ }^{2}-\mathrm{q}_{2}{ }^{2}-\mathrm{q}_{3}{ }^{2}+\mathrm{q}_{4}{ }^{2}\right)+\rho^{4} \\
& =\text { the square of the distance of the infinitesimal mass from the second }
\end{aligned}
$$

$$
\mathrm{c}=\sum_{i=0}^{\infty} c_{i} \mu^{i}, \text { the jacobi's constant, }
$$

the physical time ' $\mathrm{t}$ ' and the pseudo time 's' are connected by the differential equation

$$
\left(\frac{d t}{d s}\right)=4 r=4 \rho^{2}
$$




\section{Generating solutions}

For generating solutions, taking $\mu=0$, the reduced Hamiltonian,

$$
\mathrm{K}_{0}=1 / 2 \sum_{j=1}^{4} Q^{2}{ }_{j}+2 \rho^{2}\left(Q_{1} q_{2}-Q_{2} q_{1}+Q_{3} q_{4}-Q_{4} q_{3}+C_{0}\right)-4
$$

The Hamilton canonical equation of motion due to $\mathrm{K}_{0}$ are :

$$
\frac{d q_{j}}{d s}=\frac{\partial K_{0}}{\partial Q_{j}}, \quad \frac{d Q_{j}}{d s}=\frac{\partial K_{0}}{\partial q_{j}} \quad(j=1,2,3,4)
$$

Thus the equations of motion of an infinitesimal mass in terms of synodic KS - Variables can easily by derived from (30), (32) \& (33) as :

$$
\begin{aligned}
& \mathrm{q}_{1}^{\prime \prime}-4\left(\rho^{2}+\mathrm{q}_{1}^{2}+\mathrm{q}_{2}^{2}\right) \mathrm{q}_{2}{ }^{\prime}+4\left(\mathrm{q}_{1} \mathrm{q}_{4}-\mathrm{q}_{2} \mathrm{q}_{3}\right) \mathrm{q}_{3}{ }^{\prime}-4\left(\mathrm{q}_{1} \mathrm{q}_{3}+\mathrm{q}_{2} \mathrm{q}_{4}\right) \mathrm{q}_{4}{ }^{\prime}=4 \mathrm{q}_{1}\left(3 \rho^{4}-\mathrm{c}_{0}\right) \\
& \mathrm{q}_{2}^{\prime \prime}+4\left(\rho^{2}+\mathrm{q}_{1}^{2}+\mathrm{q}_{2}{ }^{2}\right) \mathrm{q}_{1}{ }^{\prime}+4\left(\mathrm{q}_{1} \mathrm{q}_{3}+\mathrm{q}_{2} \mathrm{q}_{4}\right) \mathrm{q}_{3}{ }^{\prime}+4\left(\mathrm{q}_{1} \mathrm{q}_{4}-\mathrm{q}_{2} \mathrm{q}_{3}\right) \mathrm{q}_{4}=4 \mathrm{q}_{2}\left(3 \rho^{4}-\mathrm{c}_{0}\right) \\
& \mathrm{q}_{3}^{\prime \prime}-4\left(\mathrm{q}_{1} \mathrm{q}_{4}-\mathrm{q}_{2} \mathrm{q}_{3}\right) \mathrm{q}_{1}^{\prime}-4\left(\mathrm{q}_{1} \mathrm{q}_{3}+\mathrm{q}_{2} \mathrm{q}_{4}\right) \mathrm{q}_{2}{ }^{\prime}-4\left(\rho^{2}+\mathrm{q}_{3}{ }^{2}+\mathrm{q}_{4}{ }^{2}\right) \mathrm{q}_{4}{ }^{\prime}=4 \mathrm{q}_{3}\left(3 \rho^{4}-\mathrm{c}_{0}\right) \\
& \mathrm{q}_{4}^{\prime \prime}+4\left(\mathrm{q}_{1} \mathrm{q}_{3}+\mathrm{q}_{2} \mathrm{q}_{4}\right) \mathrm{q}_{1}{ }^{\prime}-4\left(\mathrm{q}_{1} \mathrm{q}_{4}-\mathrm{q}_{2} \mathrm{q}_{3}\right) \mathrm{q}_{2}{ }^{\prime}+4\left(\rho^{2}+\mathrm{q}_{3}{ }^{2}+\mathrm{q}_{4}\right) \mathrm{q}_{3}{ }^{\prime}=4 \mathrm{q}_{4}\left(3 \rho^{4}-\mathrm{c}_{0}\right)
\end{aligned}
$$

By multiplying Eqs. (34) to (37) respectively by $\mathrm{q}_{1}{ }^{\prime}, \mathrm{q}_{2}{ }^{\prime}, \mathrm{q}_{3}{ }^{\prime}, \mathrm{q}_{4}{ }^{\prime}$ and integrating their sum, one has

$$
\sum_{j=1}^{4} q_{j}{ }^{2}=4 \rho^{6}-4 c_{0} \rho^{2}+8+h
$$

Here, $\mathrm{h}$ is taken as the constant of integration.

Again multiplying (34) to (37) respectively by $\mathrm{q}_{2}, \mathrm{q}_{1}, \mathrm{q}_{4} \& \mathrm{q}_{3}$ and subtracting the sum of the second and fourth from the sum of the first and third and integrating we get

$$
\mathrm{q}_{1}{ }^{\prime} \mathrm{q}_{2}-\mathrm{q}_{1} \mathrm{q}_{2}{ }^{\prime}+\mathrm{q}_{3}{ }^{\prime} \mathrm{q}_{4}-\mathrm{q}_{3} \mathrm{q}_{4}{ }^{\prime}=2 \rho^{4}+\mathrm{b}
$$

Here $b$ is the constant of integration to be determined by the initial conditions.

It is well known that in the restricted three - body problem, mean angular motion is taken to be unity i.e. $\mathrm{n}=1$ (as the total mass of the primaries is 1 and separation $=1 \Rightarrow n=1$ ) and thus the synodic KS - Variables $\mathrm{q}_{\mathrm{j}}^{\mathrm{s}}$ given in (13) will be of the form :

$$
\left.\begin{array}{ll}
\mathrm{q}_{1}=\rho \sin \left(\frac{i}{2}\right) \cos \left[\frac{(\Omega-\sigma-t)}{2}\right] & \mathrm{q}_{2}=\rho \sin \left(\frac{i}{2}\right) \sin \left[\frac{(\Omega-\sigma-t)}{2}\right] \\
\mathrm{q}_{3}=\rho \cos \left(\frac{i}{2}\right) \sin \left[\frac{(\Omega+\sigma-t)}{2}\right] & \mathrm{q}_{4}=-\rho \cos \left(\frac{i}{2}\right) \cos \left[\frac{(\Omega+\sigma-t)}{2}\right] \\
\text { and (40) one can easily find that } &
\end{array}\right\}
$$

From Eqs. (27) and (40) one can easily find that

$$
\sum_{j=1}^{4} q_{j}{ }^{\prime 2}=\rho^{\prime 2}+\rho^{2}\left(\sigma^{\prime}+16 \rho^{4}-8 \rho^{2} \sigma^{\prime} \cos i\right)
$$

And $\mathrm{q}_{1}{ }^{\prime} \mathrm{q}_{2}-\mathrm{q}_{1} \mathrm{q}_{2}{ }^{\prime}+\mathrm{q}_{3}{ }^{\prime} \mathrm{q}_{4}-\mathrm{q}_{3} \mathrm{q}_{4}{ }^{\prime}=2 \rho^{4}-\left(\frac{1}{2}\right) \rho^{2} \sigma^{\prime} \cos i$

The combinations Eqs. $(38,41)$ and $(39,42)$ yield

$$
\begin{aligned}
& \qquad \rho^{\prime 2}+\left(\frac{1}{4}\right) \rho^{2}\left(\sigma^{2}+16 \rho^{4}-8 \rho^{2} \sigma^{\prime} \cos i\right)=4 \rho^{6}-4 c_{0} \rho^{2}+8+h \\
& \text { And }-\left(\frac{1}{2}\right) \rho^{2} \sigma^{\prime} \cos i=b
\end{aligned}
$$

Following [4] and [5] at $\mathrm{s}=0, \rho=0$ and which gives $\mathrm{b}=0$, consequently $\sigma^{\prime}=0$ i.e. $\sigma=$ constant $=\sigma_{0}$ (say) and the second degree differential equation reduces to 


$$
\rho^{\prime 2}=8+h-4 c_{0} \rho^{2}
$$

At $\mathrm{h}=0$, the generating solutions with generalized period $\mathrm{s}^{*}=\mathrm{k} \frac{\pi}{2}, \mathrm{k} \in \mathrm{N}$ of the system of Eqs. (34) to (37) are given by $[6,7]$ as :

$$
\left.\begin{array}{ll}
\mathrm{q}_{1}=\rho \sin \left(\frac{i}{2}\right) \cos \left[\frac{\left(\Omega-\sigma_{0}-t\right)}{2}\right] & \mathrm{q}_{2}=\rho \sin \left(\frac{i}{2}\right) \sin \left[\frac{\left(\Omega-\sigma_{0}-t\right)}{2}\right] \\
\mathrm{q}_{3}=\rho \cos \left(\frac{i}{2}\right) \sin \left[\frac{\left(\Omega+\sigma_{0}-t\right)}{2}\right] & \mathrm{q}_{4}=-\rho \cos \left(\frac{i}{2}\right) \cos \left[\frac{\left(\Omega+\sigma_{0}-t\right)}{2}\right]
\end{array}\right\}
$$

and

$$
\begin{array}{ll}
\mathrm{Q}_{1}=\rho^{\prime} \sin \left(\frac{i}{2}\right) \cos \left[\frac{\left(\Omega-\sigma_{0}-t\right)}{2}\right] & \mathrm{Q}_{2}=\rho^{\prime} \sin \left(\frac{i}{2}\right) \sin \left[\frac{\left(\Omega-\sigma_{0}-t\right)}{2}\right] \\
\mathrm{Q}_{3}=\rho^{\prime} \cos \left(\frac{i}{2}\right) \sin \left[\frac{\left(\Omega+\sigma_{0}-t\right)}{2}\right] & \mathrm{Q}_{4}=-\rho^{\prime} \cos \left(\frac{i}{2}\right) \cos \left[\frac{\left(\Omega+\sigma_{0}-t\right)}{2}\right]
\end{array}
$$

where $\rho^{\prime}=-2 \sqrt{2} \sin \left(2 \sqrt{c_{0}} s-s_{0}\right)$

From (32)

$$
\mathrm{t}=\left(\frac{1}{c_{0}} \sqrt{c_{0}}\right)\left[4 \sqrt{\left.c_{0} s+\sin \left(4 \sqrt{c_{0}} s-2 s_{0}\right)\right]+s_{1}}\right.
$$

where $s_{1}$ is the constant of integration.

Let us introduce two variables $\theta$ and $\varphi$, where

$$
\theta=\left(\frac{\Omega-\sigma_{0}-t}{2}\right) \quad \& \quad \varphi=\left(\frac{\Omega+\sigma_{0}-t}{2}\right)
$$

Introduction of (31) yield $\theta^{\prime}=\varphi^{\prime}=-2 \rho^{2}$

$$
\begin{array}{rlrl}
\mathrm{q}_{1} & =\rho \sin \left(\frac{i}{2}\right) \cos \theta & \mathrm{q}_{2} & =\rho \sin \left(\frac{i}{2}\right) \sin \theta \\
\mathrm{q}_{3}=\rho \cos \left(\frac{i}{2}\right) \cos \varphi & \mathrm{q}_{4}=-\rho \cos \left(\frac{i}{2}\right) \cos \varphi
\end{array}
$$

and

$$
\begin{array}{ll}
\mathrm{Q}_{1}=\rho^{\prime} \sin \left(\frac{i}{2}\right) \cos \theta & \mathrm{Q}_{2}=\rho^{\prime} \sin \left(\frac{i}{2}\right) \sin \theta \\
\mathrm{Q}_{3}=\rho^{\prime} \cos \left(\frac{i}{2}\right) \cos \varphi & \mathrm{Q}_{4}=-\rho^{\prime} \cos \left(\frac{i}{2}\right) \cos \varphi
\end{array}
$$

Now from Eq.(45)

$$
\rho^{2}=8+h-4 c_{0} \rho^{2}
$$

Integrating Eq.(54) with respect to $s$ we get

$$
\mathrm{s}=\int_{0}^{\rho} \frac{d \rho}{\sqrt{8+h-4 c_{0} \rho^{2}}}
$$

with the substitution $\rho=\sqrt{(8+h) / 4 c_{0}} \sin \alpha \quad$ one can easily find $\alpha=2 \sqrt{c_{0}} s$

$$
\& \rho=\sqrt{(8+h) / 4 c_{0}} \sin 2 \sqrt{c_{0}} s
$$

Using Eqs. (58) in (35) and (55) one has

$$
\mathrm{t}=\frac{(8+h)}{8 c_{0} \sqrt{c_{0}}\left[4 \sqrt{c_{0}} s-\sin \left(4 \sqrt{c_{0}} s-2 s_{0}\right)\right]}
$$




$$
\theta=\left(\omega_{1}-\frac{t}{2}\right), \varphi=\left(\omega_{2}-\frac{t}{2}\right)
$$

where $\omega_{1}$ and $\omega_{2}$ are the constants of integration.

Thus the solutions of the problem in question at $\mu=0$ depend upon three arbitrary constants $\mathrm{h}$, $\omega_{1}$ and $\omega_{2}$.

\section{Discussion}

A system of differential equations may have infinitely many generating solutions so there are many possibilities of generating solutions of the differential Eqs. (26). Here in our case (50) represents one set of generating solution for the satellite motion.

\section{References}

[1] P. Kustaanheimo and E. Steifel, J. Reine Angew Math. 218 (1965) 204.

[2] G. Scheifle, , Cele. Mech., 2 (1970)296.

[3] E. Stiefel, and G. Scheifle, Linear and Regular Celestial Mechanics, Berlin, Heidelberg, New York,(1971).

[4] I.V. Kurcheeva, Cele. Mech, 15 (1977) 353.

[5] A. Ahmad, Cele. Mech \& Dynamical Astronomy, 61 (1995)181.

[6] G.A. Krashinaski, Bull., I.T.A , 9 (1963)205.

[7] M.R. Hassan, Bull. Astr. Soc., India, 2001. 